\title{
Treatment with zolpidem after ethanol administration potentiates the expression of ethanol-induced behavioral sensitization in mice
}

\author{
N.R.N. Brandão (iD ${ }^{1}$, M. Libarino-Santos (ii) ${ }^{1}$, E.A.V. Marinho (iD) ${ }^{1}$, T.S. Oliveira ${ }^{1}{ }^{1}$, A.L.N. Borges (ii) ${ }^{1}$, \\ A.P. Oliveira (ii) ${ }^{1}$, D. Oliveira-Campos ${ }^{1}{ }^{1}$, N. Azevedo-Souza ${ }^{1}{ }^{1}$, V.F.L. Santos ${ }^{1}{ }^{1}$, L.F. Berro ${ }^{11}{ }^{1,2}$, and \\ A.J. Oliveira-Lima ${ }^{1}{ }^{1}$ \\ ${ }^{1}$ Departamento de Ciências da Saúde, Universidade Estadual de Santa Cruz, Ilhéus, BA, Brasil \\ ${ }^{2}$ Department of Psychiatry and Human Behavior, University of Mississippi Medical Center, Jackson, MS, USA
}

\begin{abstract}
Contradictory findings suggest that the behavioral and abuse-related effects of ethanol are mediated by its action at $\alpha 1$ subunitcontaining $\mathrm{GABA}_{\mathrm{A}}\left(\alpha 1 \mathrm{GABA}_{\mathrm{A}}\right)$ receptors. In the present study, we investigated the effects of a sub-chronic post-ethanol administration treatment with zolpidem, an $\alpha 1$-preferring positive allosteric modulator at $\mathrm{GABA}_{\mathrm{A}}$ receptors, on the subsequent expression of ethanol-induced behavioral sensitization in mice. Animals received ethanol $(1.8 \mathrm{~g} / \mathrm{kg}$, ip) or saline treatments every other day for 15 days ( 8 treatment sessions) and were subsequently treated with zolpidem $(0.5 \mathrm{mg} / \mathrm{kg}$, ip) or vehicle 4 times on alternate days. At the end of the treatment phase, animals were challenged with saline or ethanol on separate days for the evaluation of the expression of conditioned locomotion and behavioral sensitization. Eight-day treatment with ethanol did not lead to the development of ethanol-induced behavioral sensitization. Animals treated with ethanol and subsequently administered vehicle showed similar locomotion frequencies during the last ethanol challenge compared to the control group receiving ethanol for the first time. Animals treated with ethanol and subsequently administered zolpidem expressed behavioral sensitization to ethanol during the ethanol challenge. The present study adds to the literature by providing further evidence of a role of $\alpha 1 G A B A_{A}$ receptors on the behavioral effects of ethanol. Because of the current highly prevalent co-abuse of ethanol and benzodiazepine drugs in humans, the use of zolpidem and other $\alpha 1 G A B A_{A}$ receptor ligands during ethanol withdrawal should be monitored carefully.
\end{abstract}

Key words: Ethanol; Behavioral sensitization; Zolpidem; Open-field; Mice

\section{Introduction}

Ethanol use disorder is a devastating disease, resulting in a series of organic, psychological, social, and economic problems. In the United States alone, nearly 17 million people suffer from ethanol-related disorders, being responsible for 88,000 deaths and costing more than $\$ 223.5$ billion annually (1). Ethanol is frequently consumed in combination with other drugs of abuse, and the combination of ethanol and sedative-hypnotic drugs, such as zolpidem, has been associated with increased likelihood of being admitted to intensive care units $(2,3)$.

Zolpidem and ethanol share similar mechanisms of action. Ethanol is a central nervous system depressant, and acts by potentiating $\gamma$-aminobutyric acid (GABA)ergic neurotransmission via action at the $\mathrm{GABA}_{\mathrm{A}}$ receptor (4). Zolpidem is an $\alpha 1$-preferring positive allosteric modulator at $\mathrm{GABA}_{\mathrm{A}}$ receptors (5). Although several subtypes of $\mathrm{GABA}_{A}$ receptors have been implicated in the abuserelated effects of ethanol (6), the role of $\alpha 1$-containing $\mathrm{GABA}_{A}\left(\alpha 1 G A B A_{A}\right)$ receptors on ethanol abuse remains controversial. Previous studies in rodents have shown a reduced preference for ethanol in $\alpha 1 G A B A_{A}$ null mutant mice (7) and reduced ethanol self-administration after treatment with $\alpha 1 G A B A_{A}$ receptor antagonists in rats (8). However, self-administration studies in non-human primates have shown little evidence of a contribution of $\alpha 1 \mathrm{GABA}_{\mathrm{A}}$ receptors on the reinforcing effects of ethanol (9), and subject-rated reinforcing effects of zolpidem were not increased by ethanol in humans (10).

In order to further elucidate the role of $\alpha 1 G A B A_{A}$ receptors on ethanol-induced behaviors, the present study was designed to investigate the effects of a sub-chronic post-ethanol administration treatment with zolpidem on

Correspondence: L.F. Berro: <lberro@umc.edu> 
the subsequent expression of ethanol-induced behavioral sensitization in mice.

\section{Material and Methods}

\section{Subjects}

Three-month-old Swiss male mice (LaBIO, Universidade Estadual de Santa Cruz, UESC) were used in the experiments. Animals were housed 10 per cage under controlled ventilation, temperature $\left(22-23^{\circ} \mathrm{C}\right)$, and lighting conditions (12 h light/dark, lights on at 6:30 am) with free access to water and food. The experiments were in accordance with the National Institute of Health Guide for Care and Use of Laboratory Animals (8th edition, revised 2011) and the Brazilian Law No. 11,794, and were approved by the Institutional Ethical Committee of UESC.

\section{Drugs}

Zolpidem $(0.5 \mathrm{mg} / \mathrm{kg})\left(\right.$ Pfizer $^{\circledR}$, USA) was dissolved in $1 \%$ Tween 80 and subsequently diluted in $0.9 \%$ saline. Ethanol $(1.8 \mathrm{~g} / \mathrm{kg})\left(\right.$ Merck $^{\mathrm{R}}$, USA) was diluted in $0.9 \%$ saline. All solutions were administered intraperitoneally (ip) at a volume of $10 \mathrm{~mL} / \mathrm{kg}$.

\section{Open-field evaluation}

Locomotor activity was measured in the open-field apparatus. The apparatus consisted of a circular wooden arena (40 cm in diameter and $50 \mathrm{~cm}$ high) with an open top and a floor divided into 19 approximately similar regions delimited by three concentric circles intersected by radial line segments. Animals were exposed to the open-field individually during 10-min sessions. Locomotor activity was tracked using the ANY-maze software (version 5.1, Stoelting, USA) and a webcam was suspended overhead.

\section{Experimental design}

Figure 1 illustrates the experimental design. Forty male mice were given a 10-min habituation session in the open-field for 3 consecutive days after a saline injection, and locomotor activity was quantified on day 3 . Animals were then allocated into 2 groups ( $N=20$ per group). The behavioral sensitization protocol ("ethanol treatment" phase) was conducted according to a protocol previously

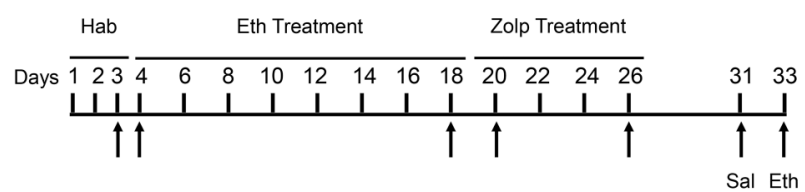

Figure 1. Experimental design. Hab: habituation. Eth treatment: ip injection every other day of saline (Sal) or ethanol (Eth, $1.8 \mathrm{~g} /$ $\mathrm{kg}$ ). Zolp treatment: ip injection every other day of vehicle or zolpidem (Zolp, $0.5 \mathrm{mg} / \mathrm{kg}$ ) in the open-field apparatus. Sal challenge: ip injection of saline. Eth challenge: ip injection of ethanol $(1.8 \mathrm{~g} / \mathrm{kg})$. Arrows indicate days in which behavior was quantified. used by our research group (11). Animals received treatments of either ethanol (Eth) or saline (Sal) every other day for 15 days ( 8 treatment sessions, days 4 to 18). Five minutes after injections, animals were individually placed in the open-field for $10 \mathrm{~min}$. Locomotor activity (distance traveled during the session) was measured on the 1st and 15 th days of this phase.

Forty-eight hours after the last ethanol session, the zolpidem treatment phase began. Mice received an ip injection of vehicle (Veh) or zolpidem (Zol) every other day for 7 days (4 sessions, days 20 to 26), forming the following groups: Sal-Veh, Eth-Veh, Sal-Zol, and Eth-Zol ( $N=10$ per group). Five minutes after each injection, mice were individually placed in the open-field for $10 \mathrm{~min}$. Locomotor activity was measured on the 1 st and last days of this phase.

Four days after the last treatment injection (day 31), all animals were acutely challenged with saline (ip) to evaluate conditioned responses in the open-field apparatus. Forty-eight hours after the saline challenge (day 33), animals were tested for drug-induced expression of behavioral sensitization to ethanol. All animals received an injection of $1.8 \mathrm{~g} / \mathrm{kg}$ ethanol and were individually placed in the open-field apparatus.

\section{Statistical analysis}

Before conducting the parametric tests, all variables were checked for normality (Shapiro-Wilk test) and homogeneity (Levene's test), which validated the use of the parametric test. Data were analyzed by one- or twoway analysis of variance (ANOVA), with or without repeated measures, or Student's $t$-test for paired samples (within-group comparisons). Post hoc comparisons were performed using Duncan's post hoc test. A P value less than 0.05 was considered to be a statistically significant difference.

\section{Results}

Analysis of the 3rd habituation session revealed no significant difference between groups (data not shown). During the ethanol treatment phase, one-way repeated measures ANOVA revealed a significant effect of treatment (Sal vs Eth) $(F(1,38)=67.38, P<0.0001)$, but not time (Day 1 vs Day 15) $(\mathrm{F}(1,38)=0.84, \mathrm{P}>0.05)$ or interaction between treatment and time $(F(1,38)=0.36, P>0.05)$. Post hoc comparisons indicated that ethanol induced hyperlocomotion (Eth > Sal, Day 1), an effect that was not sensitized after repeated ethanol administration (Day 1= Day 15) (Figure 2A).

During the zolpidem treatment phase, two-way repeated measures ANOVA revealed a significant effect of time (Day 1 vs Day 7$)(F(1,72)=17.2, P<0.0001)$, ethanol treatment (Sal vs Eth) $(\mathrm{F}(1,72)=22.07, \mathrm{P}<0.0001)$, and zolpidem treatment (Veh vs $\mathrm{Zol})(\mathrm{F}(1,72)=4.93, \mathrm{P}<0.05)$, as well as a significant interaction between time and ethanol 
treatment $(F(1,72)=5.54, P<0.05)$ and ethanol treatment and zolpidem treatment $(F(1,72)=12.43, P<0.001)$.

Post hoc comparisons showed that acute treatment with zolpidem in animals treated with saline, but not ethanol, induced hypolocomotion in mice (Sal-Zol < Sal-Veh, Day 1). Zolpidem-induced hypolocomotion was sensitized after repeated administration (Sal-Zol Day $7<$ Sal-Zol Day 1). Although ethanol-treated animals did not express conditioned locomotion to ethanol on the 1st day of the zolpidem treatment phase (Eth-Veh=Sal-Veh), the distance traveled was significantly decreased over time in ethanol-treated animals, indicating an extinction effect (Figure 2B).

During the saline challenge, two-way repeated measures ANOVA revealed no significant effect of ethanol treatment $(F(1,9)=0.5, P>0.05)$, zolpidem treatment $(F(1,9)=$ $0.15, P>0.05)$, or interaction between ethanol and zolpidem treatments $(F(1,72)=0.3, P>0.05)$ (Figure $3 A)$. Regarding the ethanol challenge, two-way repeated measures ANOVA revealed a significant interaction effect between ethanol treatment and zolpidem treatment $(F(1,9)=10.42$, $\mathrm{P}<0.05)$. Post hoc analysis showed that animals
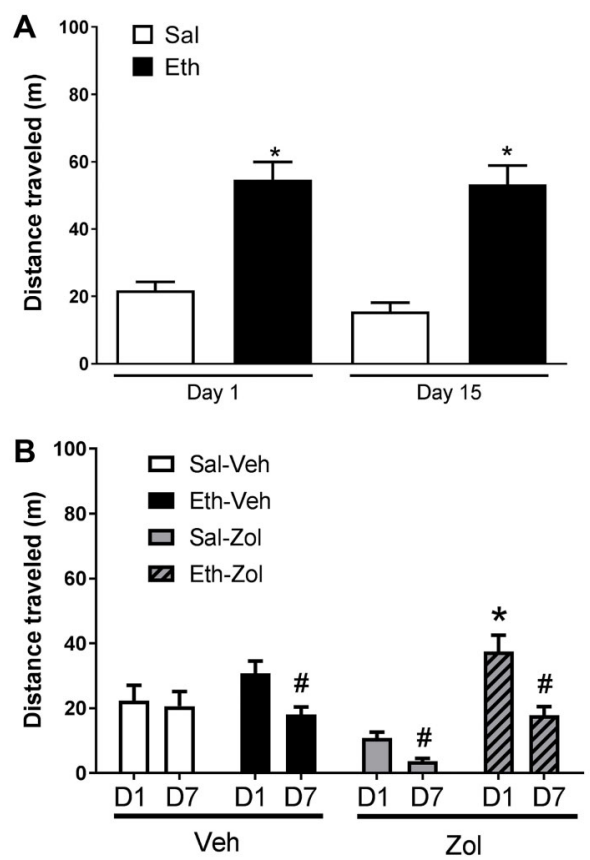

Figure 2. Locomotor activity quantification in the open-field apparatus demonstrating (A) acute hyperlocomotion induced by ethanol (Eth, $1.8 \mathrm{~g} / \mathrm{kg}$ ) (Day 1) and ethanol-induced behavioral sensitization (Day 15) after a 15-day intermittent treatment (8 ethanol injections) and (B) the behavioral effects of ip treatment with either zolpidem (Zol, $0.5 \mathrm{mg} / \mathrm{kg}$ ) or vehicle (Veh) during the post-sensitization phase for 7 intermittent days (D1 to D7). Data are reported as means $\pm S E$. ${ }^{*} P<0.05$ compared to the respective control group (A: Sal, B: Sal-Zol); ${ }^{\#} \mathrm{P}<0.05$ compared to itself on D1 (paired sample Student's $t$-test).
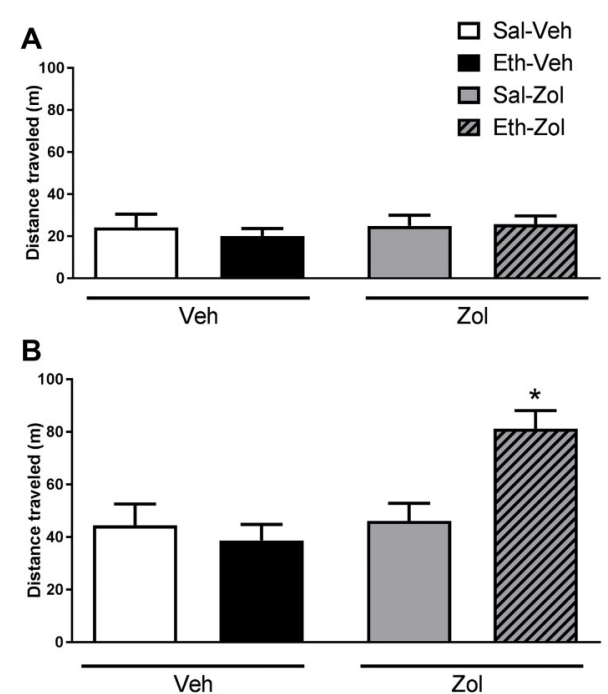

Figure 3. Locomotor activity quantification in the open-field during the (A) saline (Sal) and (B) ethanol (Eth) challenges after ethanol and/or zolpidem treatments. Data are reported as means \pm SE. ${ }^{*} \mathrm{P}<0.05$ compared to the respective control group (B: Sal-Zol) (ANOVA).

sensitized to ethanol and subsequently treated with zolpidem, but not vehicle, expressed behavioral sensitization to ethanol, with animals in the Eth-Zol group having traveled longer distances than animals in all other groups (Figure 3B).

The two-way repeated measures ANOVA considering ethanol and zolpidem treatments as factors and habituation, Days 1 and 15 of ethanol treatment, Days 1 and 7 of zolpidem treatment, and saline and ethanol challenges as repeated measures revealed individual effects of time $(F(6,252)=23.34, P<0.0001)$ and ethanol treatment $(F(1,252)=64.25, P<0.0001)$, as well as interactions between time and ethanol treatment $(F(6,252)=8.615, P<$ $0.0001)$, time and zolpidem treatment $(F(6,252)=4.456$, $\mathrm{P}<0.001)$, ethanol treatment and zolpidem treatment $(F(1,252)=7.934, P<0.01)$, and time vs ethanol treatment vs zolpidem treatment $(\mathrm{F}(6,252)=2.528, \mathrm{P}<0.05)$.

Post hoc multiple comparisons showed that the locomotor activity of the Eth-Veh group did not change across ethanol treatment/challenge days (Day 1 vs Day 15 vs ethanol challenge), indicating that this group indeed did not express locomotor activity during the ethanol challenge. On the other hand, the Eth-Zol group did show a significantly higher locomotor frequency during the ethanol challenge compared to itself during Days 1 and 15 of the ethanol treatment phase $(P=0.02$ and $P=0.0007$, respectively).

\section{Discussion}

In the present study, treatment with zolpidem after subchronic administration of ethanol (sensitization phase) promoted the expression of ethanol-induced behavioral 
sensitization. While animals in the Eth-Veh group exhibited similar locomotion frequency compared to control animals receiving ethanol for the first time (Sal-Veh), animals previously sensitized with ethanol and treated with zolpidem expressed higher locomotion frequencies than all other groups. Of note, animals receiving ethanol for the first time (Sal-Veh and Sal-Zol groups) showed similar locomotor activity levels during the ethanol challenge, suggesting that zolpidem did not simply potentiate the acute locomotor effects of ethanol and, instead, promoted the expression of ethanol-induced behavioral sensitization.

Zolpidem-induced cross sensitization was only evident during the ethanol challenge after a prolonged drug-free period. According to Lessov and Phillips (12), repeated ethanol administration associated with the test apparatus can promote modifications in neural pathways that mediate locomotor activity so that these pathways become more sensitive and responsive to a subsequent ethanol challenge. In fact, previous studies from our group have also shown that a drug-free interval is necessary for the expression of ethanol-induced behavioral sensitization (13). These neuroadaptations would explain the expression of behavioral sensitization after a drug-free period following a sensitization protocol and zolpidem treatment, and suggest that zolpidem might be modulating or further contributing to ethanol-induced neuroadaptations.

Ethanol interacts with several neurotransmitter systems $(14,15)$, and ethanol-induced activation of the mesolimbic dopaminergic pathway seems to be the main mechanism underlying acute ethanol-induced locomotor stimulation $(16,17)$. The ventral tegmental area (VTA) is one of the major regions in this pathway, and is predominantly comprised of dopamine neurons $(\sim 70 \%)$ and GABA interneurons $(\sim 20 \%)(18,19)$. Of note, ethanolinduced firing of dopamine neurons in the VTA seems to be modulated by ethanol-induced decreased firing of VTA GABAergic interneurons (20).

GABA is the main inhibitory neurotransmitter of the central nervous system, with presynaptic and post-synaptic action at inotropic $\left(G_{A B A}\right)$ and metabotropic $\left(G A B A_{B}\right)$ receptors. Each $G_{A B A}$ receptor is composed of five subunits, and the final composition of each receptor determines its distinct physiological and pharmacological properties (21). GABAergic interneurons located in the VTA selectively express $\alpha 1$-containing $\mathrm{GABA}_{\mathrm{A}}$ receptors in rats $(22,23)$. Thus, studies have focused on the investigation of the role of $\alpha 1 G A B A_{A}$ receptors on ethanol-induced behaviors, with contradictory findings. While rodent studies show favorable evidence for a potential role of $\alpha 1 \mathrm{GABA}_{\mathrm{A}}$ receptors on ethanol preference (7) and ethanol drinking (8), nonhuman primate studies show little to no evidence of a contribution of this receptor to the abuse-related effects of ethanol (9).

The present study adds to the literature by providing further evidence of a role of $\alpha 1 G A B A_{A}$ receptors in the behavioral effects of ethanol in rodents. More specifically, while 8 intermittent treatments with ethanol alone did not lead to the expression of behavioral sensitization in mice, animals treated with ethanol and subsequently administered zolpidem expressed ethanol-induced behavioral sensitization under our experimental conditions. These findings suggest that zolpidem extended the effects of ethanol when administered in the open-field apparatus. It is important to note, however, that treatment with zolpidem induced hypolocomotion in mice, indicating that the dose of zolpidem used in the present study might have induced a sedative effect in mice, opposed to the stimulant dose of ethanol. However, in the absence of zolpidem, when animals in the Zol- vs Veh-treated groups no longer showed differences in locomotor activity (saline challenge), the long-lasting effects of sub-chronic treatment with zolpidem after ethanol sensitization became evident. Our findings are in agreement with a previous study demonstrating that a 7-day treatment with zolpidem led to the development of mesolimbic dopamine-dependent neural plasticity in mice (24). It is important to note, however, that a previous study did not observe locomotor-enhancing effects of zolpidem in animals sensitized with ethanol. In the study by Linsenbardt and Boehm (25), the authors use a strain of mice known to be particularly susceptible to the development of ethanol-induced behavioral sensitization. In contrast to the present study, the authors did observe the development of behavioral sensitization to ethanol within the 15-day sensitization period. After the ethanol sensitization period, zolpidem did not potentiate the locomotor activity of mice. These data suggest that the cross-sensitization observed in the present study was, perhaps, only present because animals did not express sensitization to ethanol during the sensitization protocol in the first place.

Studies on the interaction between $\mathrm{GABA}_{\mathrm{A}}$ receptor ligands and the abuse-related effects of ethanol become extremely relevant in light of the current highly prevalent co-abuse of ethanol and benzodiazepine drugs in humans (26). Because behavioral sensitization is thought to share neuroadaptations with drug craving in humans (27), data from the present study suggest that the use of zolpidem during ethanol withdrawal, such as in the context of hypnotic drug use due to Eth withdrawal-induced sleep impairment $(28,29)$, may favor the installation of compulsive behavior.

Our findings suggested that $\alpha 1 G A B A_{A}$ receptors play an important role on ethanol-induced behaviors, and that the use of zolpidem and other $\mathrm{GABA}_{\mathrm{A}}$ receptor ligands in the context of ethanol abuse should be monitored carefully.

\section{Acknowledgments}

The authors thank Mr. José Carlos Santos de Oliveira for his capable technical support. Funding for this study was provided by Conselho Nacional de Desenvolvimento 
Científico e Tecnológico (CNPq), Fundação Coordenação de Aperfeiçoamento de Pessoal de Nível Superior

\section{References}

1. Litten RZ, Wilford BB, Falk DE, Ryan ML, Fertig JB. Potential medications for the treatment of alcohol use disorder: an evaluation of clinical efficacy and safety. Subst Abus 2016; 37: 286-298, doi: 10.1080/08897077.2015. 1133472.

2. Zosel A, Osterberg EC, Mycyk MB. Zolpidem misuse with other medications or alcohol frequently results in intensive care unit admission. Am J Ther 2011; 18: 305-308, doi: 10.1097/MJT.0b013e3181d169ed.

3. Bush DM. Emergency department visits attributed to overmedication that involved the insomnia medication zolpidem. In: The CBHSQ Report. Rockville (MD): Substance Abuse and Mental Health Services Administration (US); 2013-2014 Aug 07.

4. Grobin AC, Matthews DB, Devaud LL, Morrow AL. The role of $\operatorname{GABA}(A)$ receptors in the acute and chronic effects of ethanol. Psychopharmacology (Berl) 1998; 139: 2-19.

5. Crestani F, Martin JR, Möhler H, Rudolph U. Mechanism of action of the hypnotic zolpidem in vivo. Br J Pharmacol 2000; 131: 1251-1254, doi: 10.1038/sj.bjp.0703717.

6. Chandler CM, Overton JS, Rüedi-Bettschen D, Platt DM. $\mathrm{GABA}_{A}$ receptor subtype mechanisms and the abuse-related effects of ethanol: genetic and pharmacological evidence. Handb Exp Pharmacol 2018; 248: 615, doi: 10.1007/978-3319-96523-9.

7. Blednov $Y A$, Walker $D$, Alva $H$, Creech $K$, Findlay $G$, Harris RA. GABA $A_{A}$ receptor alpha 1 and beta 2 subunit null mutant mice: behavioral responses to ethanol. J Pharmacol Exp Ther 2003; 305: 854-863, doi: 10.1124/jpet.103.049478.

8. Harvey SC, Foster KL, McKay PF, Carroll MR, Seyoum R, Woods JE 2nd, et al. The GABA(A) receptor alpha1 subtype in the ventral pallidum regulates alcohol-seeking behaviors. J Neurosci 2002; 22: 3765-3775, doi: 10.1523/JNEUROSCI. 22-09-03765.2002.

9. Sawyer EK, Moran C, Sirbu MH, Szafir M, Van Linn M, Namjoshi $O$, et al. Little evidence of a role for the $\alpha 1 \mathrm{GABAA}$ subunit-containing receptor in a rhesus monkey model of alcohol drinking. Alcohol Clin Exp Res 2014; 38: 1108-1117, doi: 10.1111/acer.12320.

10. Wilkinson CJ. The abuse potential of zolpidem administered alone and with alcohol. Pharmacol Biochem Behav 1998; 60: 193-202, doi: 10.1016/S0091-3057(97)00584-4.

11. Oliveira-Lima AJ, Santos R, Hollais AW, Gerardi-Junior CA, Baldaia MA, Wuo-Silva R, et al. Effects of ayahuasca on the development of ethanol-induced behavioral sensitization and on a post-sensitization treatment in mice. Physiol Behav 2015; 142: 28-36, doi: 10.1016/j.physbeh.2015.01.032.

12. Lessov CN, Phillips TJ. Duration of sensitization to the locomotor stimulant effects of ethanol in mice. Psychopharmacology (Berl) 1998; 135: 374-382.

13. Libarino-Santos $M$, de Santana Santos ACG, Cata-Preta EG, Barros-Santos T, Nunes Brandão NR, Borges ALN,
(CAPES), and Fundação de Amparo a Pesquisa do Estado da Bahia (FAPESB).

et al. Role of the treatment environment in the effects of aripiprazole on ethanol-induced behavioral sensitization and conditioned place preference in female mice. Drug Alcohol Depend 2020; 208: 107856, doi: 10.1016/j.drugalcdep.2020. 107856.

14. Broadbent J, Kampmueller KM, Koonse SA. Expression of behavioral sensitization to ethanol by DBA/2J mice: the role of NMDA and non-NMDA glutamate receptors. Psychopharmacology (Berl) 2003; 167: 225-234.

15. Vengeliene V, Bilbao A, Molander A, Spanagel R. Neuropharmacology of alcohol addiction. Br J Pharmacol 2008; 154: 299-315, doi: 10.1038/bjp.2008.30.

16. Ron D, Jurd R. The "ups and downs" of signaling cascades in addiction. Sci STKE 2005; 2005: re14.

17. Stahl SM. Stahl's essential psychopharmacology: neuroscientific basis and practical applications. New York: Cambridge University Press; 2013.

18. Yamaguchi T, Sheen W, Morales M. Glutamatergic neurons are present in the rat ventral tegmental area. Eur J Neurosci 2007; 25: 106-118, doi: 10.1111/j.1460-9568.2006.05263.x.

19. Dobi A, Margolis EB, Wang HL, Harvey BK, Morales M. Glutamatergic and nonglutamatergic neurons of the ventral tegmental area establish local synaptic contacts with dopaminergic and nondopaminergic neurons. J Neurosci 2010; 30: 218-229, doi: 10.1523/JNEUROSCI.388409.2010.

20. Gallegos RA, Lee RS, Criado JR, Henriksen SJ, Steffensen SC. Adaptive responses of gamma-aminobutyric acid neurons in the ventral tegmental area to chronic ethanol. J Pharmacol Exp Ther 1999; 291: 1045-1053.

21. Olsen RW, Sieghart W. International Union of Pharmacology. LXX. Subtypes of gamma-aminobutyric acid(A) receptors: classification on the basis of subunit composition, pharmacology, and function, Update. Pharmacol Rev 2008; 60: 243-260, doi: 10.1124/pr.108.00505.

22. Gao B, Fritschy JM. Selective allocation of GABAA receptors containing the alpha 1 subunit to neurochemically distinct subpopulations of rat hippocampal interneurons. Eur J Neurosci 1994; 6: 837-853, doi: 10.1111/j.1460-9568. 1994.tb00994.x.

23. Gao B, Fritschy JM, Benke D, Mohler H. Neuron-specific expression of GABAA-receptor subtypes: differential association of the alpha 1- and alpha 3-subunits with serotonergic and GABAergic neurons. Neuroscience 1993; 54: 881892, doi: 10.1016/0306-4522(93)90582-Z.

24. Shibasaki M, Tsuyuki T, Ando K, Otokozawa A, Udagawa $Y$, Watanabe K, et al. Implication of KCC2 in the sensitization to morphine by chronic ethanol treatment in mice. Synapse 2014; 68: 39-43, doi: 10.1002/syn.21688.

25. Linsenbardt DN, Boehm SL 2nd. Ethanol-induced locomotor sensitization in DBA/2J mice is associated with alterations in $\operatorname{GABA}(\mathrm{A})$ subunit gene expression and behavioral sensitivity 
to GABA(A) acting drugs. Pharmacol Biochem Behav 2010; 95: 359-366, doi: 10.1016/j.pbb.2010.02.014.

26. Morel A, Grall-Bronnec M, Bulteau S, Chauvin-Grelier P, Gailledrat L, Pinot ML, et al. Benzodiazepine dependence in subjects with alcohol use disorders: what prevalence? Expert Opin Drug Saf 2016; 15: 1313-1319, doi: 10.1080/ 14740338.2016.1221922.

27. Robinson TE, Berridge KC. The neural basis of drug craving: an incentive-sensitization theory of addiction. Brain
Res Brain Res Rev 1993; 18: 247-291, doi: 10.1016/01650173(93)90013-P.

28. Mendelson WB, Majchrowicz E, Mirmirani N, Dawson S, Gillin JC, Wyatt RJ. Sleep during chronic ethanol administration and withdrawal in rats. J Stud Alcohol 1978; 39: 1213-1223, doi: 10.15288/jsa.1978.39.1213.

29. Trevisan LA, Boutros N, Petrakis IL, Krystal JH. Complications of alcohol withdrawal: pathophysiological insights. Alcohol Health Res World 1998; 22: 61-66. 